Article

\title{
Hypolipidemic Activities of Two Pentapeptides (VIAPW and IRWWW) from Miiuy Croaker (Miichthys miiuy) Muscle on Lipid Accumulation in HepG2 Cells through Regulation of AMPK Pathway
}

\author{
Yu-Mei Wang ${ }^{1}$, Xin Pan ${ }^{2}$, Yu He ${ }^{1}$, Chang-Feng Chi ${ }^{2, *}$ and Bin Wang ${ }^{1, *}$ \\ 1 Zhejiang Provincial Engineering Technology Research Center of Marine Biomedical Products, \\ School of Food and Pharmacy, Zhejiang Ocean University, Zhoushan 316022, China; \\ wangym731@126.com (Y.-M.W.); heyu19950618@163.com (Y.H.) \\ 2 National and Provincial Joint Laboratory of Exploration and Utilization of Marine Aquatic Genetic \\ Resources, National Engineering Research Center of Marine Facilities Aquaculture, School of Marine Science \\ and Technology, Zhejiang Ocean University, Zhoushan 316022, China; Uniquepan2015@163.com \\ * Correspondence: chichangfeng@hotmail.com (C.-F.C.); wangbin4159@hotmail.com (B.W.); \\ Tel./Fax: +86-580-255-4818 (C.-F.C.); +86-580-255-4781 (B.W.)
}

Received: 24 December 2019; Accepted: 22 January 2020; Published: 23 January 2020

\begin{abstract}
In this work, the hypolipidemic activities of two pentapeptides (VIAPW and IRWWW) from miiuy croaker (Miichthys miiuy) muscle on oleic acid (OA)-induced lipid accumulation in HepG2 cells were investigated. VIAPW and IRWWW could significantly inhibit lipid accumulation induced by OA and decreased intracellular levels of intracellular triglyceride (TG) and total cholesterol (TC) in a dose-effect dependence manner. At the concentration of $100 \mu \mathrm{m}$, the TG levels of VIAPW $(0.201 \pm 0.006 \mathrm{~mm})$ and IRWWW $(0.186 \pm 0.005 \mathrm{~mm})$ were very $(p<0.01)$ and extremely $(p<0.001)$ significantly lower than those $(0.247 \pm 0.004 \mathrm{~mm})$ of the OA model group; the levels of TC of VIAPW $(45.88 \pm 0.74 \mu \mathrm{g} / \mathrm{mg}$ protein) and IRWWW $(41.02 \pm 0.14 \mu \mathrm{g} / \mathrm{mg}$ protein) were very $(p<0.01)$ and extremely $(p<0.001)$ significantly lower than that $(53.45 \pm 0.10 \mu \mathrm{g} / \mathrm{mg}$ protein) of the OA model group $(p<0.01)$. The hypolipidemic mechanisms of VIAPW and IRWWW were to down-regulate the expression levels of genes of SREBP-1c, SREBP-2, FAS, ACC, and HMGR in lipid synthesis and to up-regulate the expression levels of genes of PPAR $\alpha$, ACOX-1, and CPT- 1 in lipid oxidation. These results suggested that VIAPW and IRWWW could play their hypolipidemic activities in HepG2 cells through regulation of AMPK pathway and act as hypolipidemic nutrient ingredients applied in public healthy and functional foods.
\end{abstract}

Keywords: miiuy croaker (Miichthys miiuy); muscle; VIAPW; IRWWW; hypolipidemic activity

\section{Introduction}

Hyperlipidemia is referring to the abnormally high levels of total triglycerides (TG), cholesterol (TC), and lipoproteins in the blood circulation, which is a key contributor to atherosclerosis, coronary artery disease (CAD), acute pancreatitis, and peripheral vascular disease (PVD) [1,2]. Hyperlipidemia is the best-known disease in connection with a sedentary lifestyle, inherited genetic disorder, high-fat diets, diabetes, and obesity [3]. In recent years, the population of hyperlipidemia has been increasing gradually around the world due to the unhealthy lifestyles, such as smoking, high fat acid (FA) and sugar diets, and physical inactivity [4]. Therefore, synthetic drugs including bile acid binding resins, nicotinic acid derivatives, and cholesterol absorption inhibitors were applied to regulate the lipid levels of plasma [5]; however, their potential toxicities cause great trouble to the patient and limit their 
long-term applications [6]. In addition, functional foods with abundant hypolipidemic substances enjoy great popularity for the treatment of moderate hyperlipidemia [6,7]. Therefore, searching for efficient ingredients from natural sources to produce lipid-lowering medicines or health products attracts continuing concern [8].

To date, active ingredients from natural resources including polysaccharides, quinones, alkaloids, flavonoids, steroidal saponins, and proteins and peptides, have been identified as potential hypolipidemic agents because they could accelerate the catabolism of exogenous lipid, suppressing the biosynthesis of endogenous lipids and inhibiting lipid absorption and peroxidation [4,8-10]. Among them, food-derived peptides attract extensive attention because they have physiological functions beyond their high nutritional values and can act as drugs and functional products [11-14]. Fan et al. (2018) purified and identified four peptides including NPVWKRK, NALKCCHSCPA, CANPHELPNK, and LNNPSVCDCDCMMKAAR from protein hydrolysate of Spirulina platensis. Among them, NPVWKRK and CANPHELPNK could significantly decrease the accumulation of TG dropping to $23.7 \%$ and $19.5 \%$ at a concentration of $600 \mu \mathrm{g} / \mathrm{mL}$, respectively $(p<0.05)$ [15]. LPYP, IAVPGEVA, and IAVPTGVA from digesting glycinin could improve low-density lipoprotein (LDL) uptake through activating the LDLR-sterol regulatory element-binding protein (SREBP) pathway and act as a competitive inhibitor of 3-hydroxy-3-methylglutaryl CoA reductase (HMGR) to inhibit the biosynthesis of cholesterol $[7,16]$. IIAEK from a milk $\beta$-lactoglobulin hydrolysate could significantly affect the level of serum cholesterol and display a greater cholesterol-lowering capacity in animal experiments [17]. Lee et al. reported that Glu-Phe (EF) isolated from onion could control the expression of SREBP-1c and its lipogenic target genes to decrease the lipid accumulation in mouse hepatocytes [18]. In an ovariectomized rats model, chickpea peptides (ChPs) decreased the adipose tissue size, body weight, TC, TG, LDL, cholesterol, liver TC and TG, and serum atherogenic index [19]. The mechanism was that ChPs could inhibit the activities of HMGR and FA synthetase (FAS), down-regulate the expression of SREBP-1c and peroxisome proliferator-activated receptors (PPAR) $\gamma$, and up-regulate the expression of estrogen receptor (ER) $\alpha, E R \beta$, and liver $\mathrm{X}$ receptor (LXR) $\alpha$. Therefore, food-derived peptides play an important role in controlling lipid metabolism and decreasing body FA and may be more appealing to eliminate the side-effects of hyperlipidemia.

In recent years, seafood-derived peptides with antihypertensive, antioxidant, and antitumor activities have been characterized from many marine products and their by-products [10,11,20-22]. In contrast, little attention has been paid to peptides with hypolepidemic, anti-obesity, antiatherogenic and cardioprotective effects [2]. In our previous report, ten antioxidant pentapeptides separated from the hydrolysate of miiuy croaker muscle were determined as YASVV (M1), NFWWP (M2), FWKVV (M3), TWKVV (M4), FMPLH (M5), YFLWP (M6), VIAPW (M7), WVWWW (M8), MWKVW (M9), and IRWWW (M10) [23]. Furthermore, those peptides, especially VIAPW (M7) and IRWWW (M10), showed inhibitory activity on lipid accumulation in HepG2 cells. Therefore, the objective of this study was to investigate the hypolipidemic mechanisms of VIAPW and IRWWW in a hyperlipidemia HepG2 cells model induced by oleic acid (OA) for their application in functional foods.

\section{Materials and Methods}

\subsection{Materials}

HepG2 cells were purchased from the China Cell Bank of the Institute of Biochemistry and Cell Biology (Shanghai, China). Dulbecco's Modified Eagle's medium (DMEM) and Roswell Park Memorial Institute (RPMI)-1640 medium were purchased from Gino biotechnology Co., Ltd. (Hangzhou, China). Methylthiazolyldiphenyl-tetrazolium bromide (MTT), OA, simvastatin (SV), and $\beta$-actin were purchased from Sigma-Aldrich (Shanghai) Trading Co., Ltd. (China). Paraformaldehyde (4\%) and lipofectamine 2000 were purchased from Thermo Fisher Scientific Co. Ltd. (Shanghai, China). PrimeScript RT reagent Kit with genomic DNA (gDNA) Eraser and $2 \times$ KAPA Master Mix were purchased from Takara Biomedical Technology (Beijing) Co. Ltd. (China). FA free bovine serum 
albumin (BSA) was purchased from Millipore Co. Ltd. (Shanghai, China). Bicinchoninic acid (BCA) protein assay kit and L-Glutamine were purchased from Beijing Solarbio Co. Ltd. (China). TransStart Top Green quantitative PCR (qPCR) superMix kit was purchased from TransGen Biotech Co. Ltd. (Beijing, China). SREBP-1c rabbit polyclonal antibody was purchased from Abcom Co. Ltd. (Maharashtra, India). Peptides (M1-M10) were chemically synthetized using a solid phase peptide synthesis method (synthetized by China Peptides Co. Ltd.) according to the amino acid sequences reported by He et al. [23]. The purity of the synthesized peptides was higher than $98 \%$ as analyzed via reversed-phase high-performance liquid chromatography (RP-HPLC) method. The molecular mass of the synthesized peptides was confirmed by the manufacturer using electrospray ionization mass spectrometry (ESI-MS).

\subsection{HepG2 Cell Culture}

HepG2 cells were cultured in DMEM (containing $2 \mathrm{~mm}$ glutamine, $50 \mathrm{U} / \mathrm{mL}$ penicillin, $50 \mathrm{mg} / \mathrm{mL}$ streptomycin, and $10 \%$ fetal bovine serum (FCS)). Cells were incubated in a humidified atmosphere of $5 \% \mathrm{CO}_{2}$ at $37^{\circ} \mathrm{C}$. When the monolayer reached about $70 \%$ confluent, the media were replaced with serum-free DMEM for $24 \mathrm{~h}$ ahead of experiments. After that, HepG2 cells were added to samples at designed concentrations in serum-free media for $24 \mathrm{~h}$. SV $(1 \mathrm{~mm})$ served as a positive control, and the control group without samples was prepared distilled water.

\subsection{Cell Viability Assay}

The viability assay of HepG2 cells was evaluated using an MTT assay by a previous method [12,24]. In brief, $100 \mu \mathrm{L}$ of peptides (M1-M10) at a concentration of $100 \mu \mathrm{m} \mathrm{s}$ and $100 \mu \mathrm{L}$ of growth media were added to the HepG2 cells. The control cultures were treated with the extracting solution without the peptides. In addition, the blank wells contained $100 \mu \mathrm{L}$ of growth medium without HepG2 cells. Cell viability (\% of control) was measured after incubating for $24 \mathrm{~h}$.

\subsection{Oil Red O Staining Assay}

The assay was performed using an Oil Red O staining kit (O0625) by Sigma-Aldrich (Shanghai) Trading Co. Ltd. (Shanghai, China) according to the manufacturer's instructions [24]. HepG2 cells were fixed on 96 well plates with $4 \%$ formaldehyde for $30 \mathrm{~min}$ and washed with phosphate buffered saline (PBS) twice. After rinsing with $60 \%$ isopropanol for $10 \mathrm{~min}$, isopropanol was removed. HepG2 cells were incubated in 3\% Oil Red O solution for $1 \mathrm{~h}$ and rinsed with PBS thrice to wipe off the unbound dye. Finally, stained cells were dissolved in DMSO, and the ultraviolet absorbance at $358 \mathrm{~nm}$ was determined. Images of stained cells were taken using the inverted microscope Olympus IX71 (Olympus Co. Ltd., Shinjuku, Japan).

\subsection{Preparation of Protein Extract of HepG2 Cells}

HepG2 cells were washed twice using PBS and treated with lysis buffer (1\% deoxycholate, $1 \%$ Triton X-100, 0.1\% SDS) for $20 \mathrm{~min}$ on ice. After that, the solution was centrifuged at $12,000 \times g$ at $4{ }^{\circ} \mathrm{C}$ for $20 \mathrm{~min}$. Protein concentration was measured by a BCA protein assay kit according to the manufacturer's instructions (Beijing Solarbio Co. Ltd., Beijing, China).

\subsection{Cellular TC and TG Contents Analysis}

Cellular TC and TG contents were determined according to the previous method [24]. In brief, HepG2 cells were seeded in 6-well-plates and treated under the designed processes. Then, cell lysates were prepared according to the above method. TC and TG contents were measured using the TC (BioVison K622-100) and TG (BioVison K603-100) assay kits following the manufacturer's instructions (Shanghai Yubo Bioengineering Co. Ltd., China). 


\subsection{Fluorescence Quantitative Polymerase Chain Reaction (PCR) Analysis}

RNA extraction: HepG2 cells $\left(2.5 \times 10^{5}\right.$ cells/well $)$ in 12 -well plates $(1 \mathrm{~mL})$ were incubated in DMEM (containing 10\% FCS) for two days. The HepG2 cells were washed twice using PBS and incubated in $500 \mu \mathrm{L}$ of FCS-free DMEM (containing 1.0\% (w/v)) BSA for two days with or without $0.75 \mathrm{~mm}$ sodium oleate or sodium oleate plus samples $(100 \mu \mathrm{g} / \mathrm{mL})$. After culture, HepG2 cells culture plates were placed on ice, cell culture media were wiped off, and plates were rinsed with ice-cold Tris-buffered saline (TBS). Treated HepG2 cells from each disc were lysed in TBS $(150 \mu \mathrm{L}$, containing $0.02 \%$ Triton-X100 detergent) and were moved to $1.5 \mathrm{~mL}$ tubes. To facilitate cell lysis, the tubes with HepG2 cells were frozen using liquid nitrogen and thawed quickly in a $37^{\circ} \mathrm{C}$ water bath; this process of cell lysis was repeated twice. Total ribonucleic acid (RNA) was separated from the lysed cells using a TRIzol (Thermo Fisher Scientific (China) Co. Ltd., Shanghai, China). The total amount and purity of RNA were measured using a NanoDrop 2000/2000 c Spectrophotometer (Thermo Fisher Scientific (China) Co. Ltd., Shanghai, China). RNA was reverse transcribed into complementary DNA (cDNA) using a TransStart Top Green qPCR superMix kit with fluorescence labeling of Sybr Green according to manufacturer's instructions (TransGen Biotech Co. Ltd., Beijing, China). Amplification was carried out using a three-step temperature cycle in a $10 \mu \mathrm{L}$ reaction system as follows: pre-degenerated at $95^{\circ} \mathrm{C}$ for $1 \mathrm{~min}$, degenerated at $95^{\circ} \mathrm{C}$ for $20 \mathrm{~s}$, renaturated at $58^{\circ} \mathrm{C}$ for $30 \mathrm{~s}$, and extended at $72{ }^{\circ} \mathrm{C}$ for $10 \mathrm{~s}$ with 39 cycles. The specificity of PCR was verified by melting curve analysis from $72{ }^{\circ} \mathrm{C}$ to $95^{\circ} \mathrm{C}$. The GADPH gene was chosen as the internal reference while the threshold and $\mathrm{Ct}$ (threshold cycle) values acquired via real-time PCR (RT PCR) were used to analyze the genes' mRNA levels according to the $2^{-\Delta \Delta C t}$ method. All the data were normalized and are presented as mean \pm standard error (SE) $(n=3)$. The data were processed in the Statistical Product and Service Solutions (SPSS) software. The forward and reverse PCR primer sequences were as follows in Table 1.

Table 1. The primer sequences of gene amplification.

\begin{tabular}{cc}
\hline Primer & Sequence $\mathbf{( 5}^{\prime}-\mathbf{3}^{\prime} \mathbf{)}$ \\
\hline ACC-F & TGATGTCAATCTCCCCGCAGC \\
ACC-R & TTGCTTCTTCTCTGTTTTCTCCCC \\
SREBP-1c-F & CCATGGATGCACTTTCGAA \\
SREBP-1c-R & CCAGCATAGGGTGGGTCAA \\
SREBP-2-F & CTGCAACAACAGACGGTAATGA \\
SREBP-2-R & CCATTGGCCGTTTGTGTCAG \\
FAS-F & CGGTACGCGACGGCTGCCTG \\
FAS-R & GCTGCTCCACGAACTCAAACACCG \\
HMGR-F & GGACCCCTTTGCTTAGATGAAA \\
HMGR-R & CCACCAAGACCTATTGCTCTG \\
CPT1-F & CGTCTTTTGGGATCCACGATT \\
CPT1-R & TGTGCTGGATGGTGTCTGTCTC \\
ACOX1-F & GGGCATGGCTATTCTCATTGC \\
ACOX1-R & CGAACAAGGTCAACAGAAGTTAGGTTC \\
PPAR $\alpha-\mathrm{F}$ & AAAAGCCTAAGGAAACCGTTCTG \\
PPAR $\alpha-\mathrm{R}$ & TATCGTCCGGGTGGTTGCT \\
\hline
\end{tabular}

\subsection{Western Blot Assay}

Western blot assay was performed according to the previous method [25]. HepG2 cells at a density of $1 \times 10^{5}$ cells/well were seeded in six-well plates for one day and then treated with $3 \mathrm{mg} / \mathrm{mL}$ of samples for $24 \mathrm{~h}$; the cell culture medium was used as a negative control. After that, $1 \times 10^{5}$ cells were gathered using centrifugation at $9000 \times g$ for $5 \mathrm{~min}$ at $4{ }^{\circ} \mathrm{C}$ and rinsed twice with cold PBS $(\mathrm{pH}$ 7.2). The cells collected from the six-well plates were handled with lysis buffer ( $200 \mu \mathrm{L}$, containing phenylmethanesulfonylfluoride fluoride) for $0.5 \mathrm{~h}$. Then, the HepG2 cells were centrifuged at $12,000 \times g$ for $5 \mathrm{~min}$, and the protein contents in supernatant were determined using BCA assay and separated 
using sodium dodecyl sulfate-polyacrylamide gel electrophoresis (SDS-PAGE). After that, the proteins in resolving gel were transferred to a polyvinylidene difluoride membrane, and the membrane was blocked with $10 \%$ non-immune serum for $2 \mathrm{~h}$ and then incubated with rabbit monoclonal antibody (1:1000) for $12 \mathrm{~h}$ at $4{ }^{\circ} \mathrm{C}$. After rinsing thrice using TBS containing $0.1 \%$ Tween-20 (TBST) buffer, the membrane was incubated with another antibody (goat-anti-rabbit horseradish peroxidase [HRP]-conjugated 1:3000) at $25^{\circ} \mathrm{C}$ for $2 \mathrm{~h}$ and then rinsed with TBST. The intensity of the specific immunoreactive bands was measured using enhanced chemiluminescence, quantified by densitometry, and expressed as a ratio to $\beta$-actin.

\subsection{Statistical Analysis}

All assays were carried out in triplicate and the resulted data were expressed as means \pm standard deviation (SD, $n=3$ ). ANOVA test using SPSS 19.0 was applied to analyze the experimental data. Significant differences among the parameters means were determined by using Duncan's multiple range test.

\section{Results and Discussion}

\subsection{Effects of Peptides (M1-M10) on HepG2 Cell Viability}

HepG2 cells have high differentiation and show many characteristics of genotypes of normal human liver hepatocytes [26]. In consequence, HepG2 cells are an ideal model system applied for studying human liver metabolism in vitro, screening the cytotoxicity capacity of novel functional molecules at the lead generation stage, and detecting environmental and dietary cytotoxins and genotoxins $[27,28]$.

Cell viability determines the response of cells to stress stimuli and usually is used to look at the effects of pharmacological compounds on cells. The viabilities of HepG2 cells treated with peptides YASVV (M1), NFWWP (M2), FWKVV (M3), TWKVV (M4), FMPLH (M5), YFLWP (M6), VIAPW (M7), WVWWW (M8), MWKVW (M9), and IRWWW (M10) were presented in Figure 1, the viability of cells treated with peptide TWKVV (M4) was significantly weaker than that of the normal control group at $100 \mu \mathrm{m}$ for $24 \mathrm{~h}(p<0.05)$. Furthermore, the viability of cells treated with peptide YFLWP (M6) was very significantly weaker than that of normal control group at the same concentration $(p<0.01)$. Therefore, TWKVV (M4) and YFLWP (M6) have stronger cytotoxic activity to HepG2 cells than the other eight peptides and are unfit for serving as the candidate molecules of non-anti-tumor functional food and/or drugs. Therefore, all test peptide except TWKVV (M4) and YFLWP (M6) did not significantly affect the normal proliferation of HepG2 cells and could be applied for the development of non-tumor drugs and functional food.

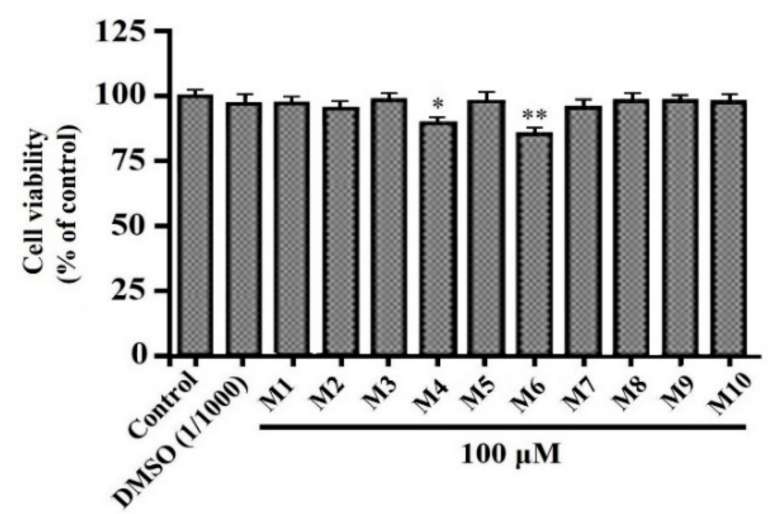

Figure 1. Effects of antioxidant peptides (M1-M10) from miiuy croaker (Miichthys miiuy) muscle on viabilities of HepG2 cells at $100 \mu \mathrm{m}$. Means \pm SD $(n=3)$ are used to express the experiment data. ** $p<0.01, * p<0.05$ versus normal control group. 


\subsection{Effects of VIAPW (M7) and IRWWW (M10) on Lipid Accumulation}

The effects of antioxidant peptides (M1-M10) on lipid accumulation in HepG2 cells pretreated with OA were studied. As shown in Figure 2, the intracellular lipid content of the model group pre-treatment with $\mathrm{OA}$ at $80 \mu \mathrm{m}$ after $24 \mathrm{~h}$ was dramatically higher than the lipid content of the normal control group $(p<0.01)$, which indicated that the OA cell model was successfully established. In comparison with the OA cell model group, VIAPW (M7) could significantly decrease the lipid accumulation in HepG2 cells at $100 \mu \mathrm{m}(p<0.05)$, and IRWWW (M10) and the positive control of SV could very significantly reduce the lipid accumulation in HepG2 cells at same concentration $(p<0.01)$. To directly observe the effects of VIAPW (M7) and IRWWW (M10) on the lipid accumulation of HepG2 cells, the tested HepG2 cells were dyed using Oil Red O, and their images were taken and shown in Figure 3. In comparison with the normal control group, higher cellular lipid content in the cell model group pre-treatment with OA was observed. In addition, more lipid globules inside cells were also found. However, the images (Figure 3) of VIAPW (M7)-, IRWWW (M10)-, and SV-treated cell groups (especially IRWWW (M10) and SV) were clearly different from that of the model cell group, which illustrated that they could dispose of most of the intracellular lipid.

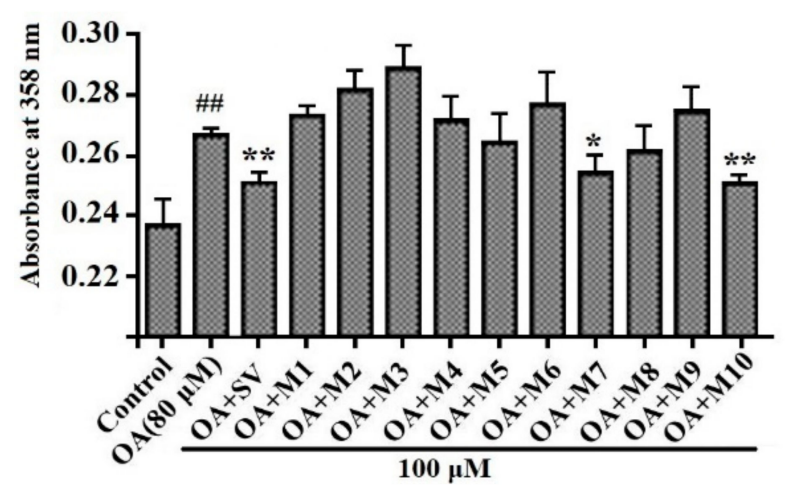

Figure 2. Effects of antioxidant peptides (M1-M10) from miiuy croaker (M. miiuy) muscle on lipid accumulation of HepG2 cells at $100 \mu \mathrm{m}$. Means \pm SD $(\mathrm{n}=3)$ are used to express the experiment data. \#\# $p<0.001$ versus control group; ${ }^{* *} p<0.01,{ }^{*} p<0.05$ versus oleic acid (OA) model group.

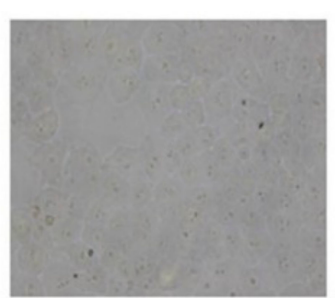

Control

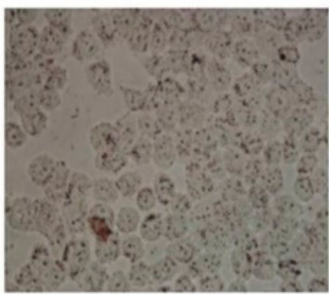

Positive control (SV)

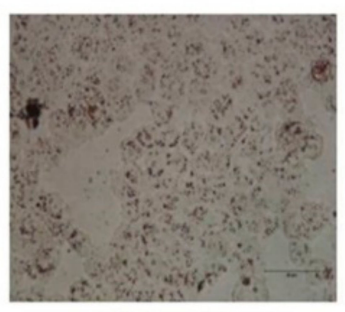

VIAPW (M7)

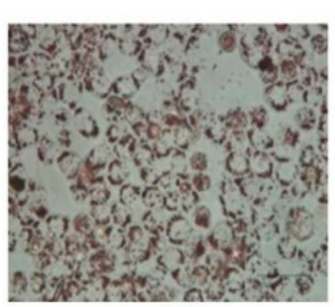

OA

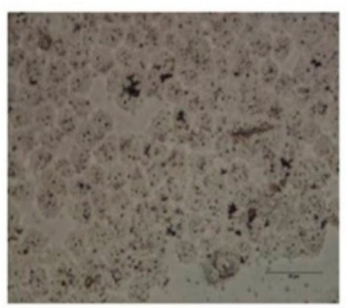

IRWWW (M10)

Figure 3. Effects of VIAPW (M7) an, IRWWW (M10) from miiuy croaker (M. miiuy) muscle at the concentration of $100 \mu \mathrm{m}$ on morphological characteristics of HepG2 cells for $24 \mathrm{~h}$ compared to normal and positive control (simvastatin (SV)) groups. The images were taken using an inverted microscope $(\times 400)$. 
Excess lipid accumulated in the hepatic cells, which acts as an indicator of a lipid overload and a mediator of metabolic syndrome. Moreover, the steatosis may translate into nonalcoholic steatohepatitis (NASH), cirrhosis, and hepatocellular carcinoma with a range of cell histological alterations [29,30]. Hence, VIAPW (M7) and IRWWW (M10) might be good lipid-lowering candidates, and their mechanisms were studied more profoundly and meticulously in the following experiments.

\subsection{Effects of VIAPW (M7) and IRWWW (M10) on Lowering Intracellular TG and TC Contents}

TG and TC are separate types of lipids that circulate in blood. TG is a major source of energy for the body through storing unused calories in adipocytes and muscle cells. TC is a ubiquitous constituent of cell membranes. High TG levels are harmful and increase the risk of heart disease, stroke, acute pancreatitis, obesity, and metabolic syndrome [31]. High TC levels are one of the dangerous factors that can result in atherosclerotic cardiovascular disease (ASCVD), including heart attack, transient ischemic attack, stroke, and peripheral artery disease [32].

Effects of VIAPW (M7) and IRWWW (M10) on the cellular TG and TC contents were presented in Figure 4. The TC and TG contents in OA cell model group were $0.247 \pm 0.004 \mathrm{~mm}$ and $53.45 \pm 0.15 \mu \mathrm{g} / \mathrm{mg}$ protein, respectively, which were extremely $(p<0.001)$ significantly higher than those of the blank control group (TC content: $0.144 \pm 0.002 \mathrm{~mm}$, and TG content: $42.11 \pm 0.32 \mu \mathrm{g} / \mathrm{mg}$ protein), respectively. The data manifested that the hyperlipidemic cell model was successfully built. After treatment with VIAPW (M7) and IRWWW (M10) at the concentrations of 10, 50, and $100 \mu \mathrm{m}$, the TC and TG contents in hyperlipidemic HepG2 cell model decreased in a concentration-effect relationship, and the TG and TC levels of VIAPW (M7) treated group were lower than those of IRWWW (M10) group at all the tested concentrations. In addition, Figure 4A indicated that the TG levels of VIAPW (M7) and IRWWW (M10) groups were significantly less than those of the OA model and the negative control groups at $50 \mu \mathrm{m}(p<0.05)$, but the TG levels of VIAPW (M7) and IRWWW (M10) groups were very $(p<0.01)$ and extremely $(p<0.001)$ less than those of the OA model and negative control groups at $100 \mu \mathrm{m}$. Figure 4B illustrates that the TC level of VIAPW (M7) was very significantly lower than that of the OA model and negative control groups at $100 \mu \mathrm{m}(p<0.01)$, but the TC levels of the IRWWW (M10) groups were very $(p<0.01)$ and extremely $(p<0.001)$ lower than those of the OA model and negative control groups at 50 and $100 \mu \mathrm{m}$, respectively. Those results indicate that VIAPW (M7) and IRWWW (M10) could inhibit the synthesis of TC and TG and activate the catabolism of these compounds. In addition, the ability of IRWWW (M10) to lower the TG and TC levels was higher than that of VIAPW (M7) group at the same concentrations. In addition, the TG and TC levels of IRWWW (M10) groups at $100 \mu \mathrm{m}$ were extremely $(p<0.001)$ less than those of the OA model group, which were close to the levels of SV group at $10 \mu \mathrm{m}$. Simvastatin (SV) is a statin drug and used to decrease the risk of cardiovascular diseases through lowering the cholesterol content by inhibiting the activity of HMGR. However, some side effects including indigestion, diarrhoea, nausea, muscle aches or pains, and weakness affect the patient's application. In our future study, we will increase the biological activities (bioavailability) of VIAPW (M7) and IRWWW (M10) through modifying their chemical structures. In addition, the toxic side effects of VIAPW (M7) and IRWWW (M10) and their derivatives will also be the focus of our attention. 

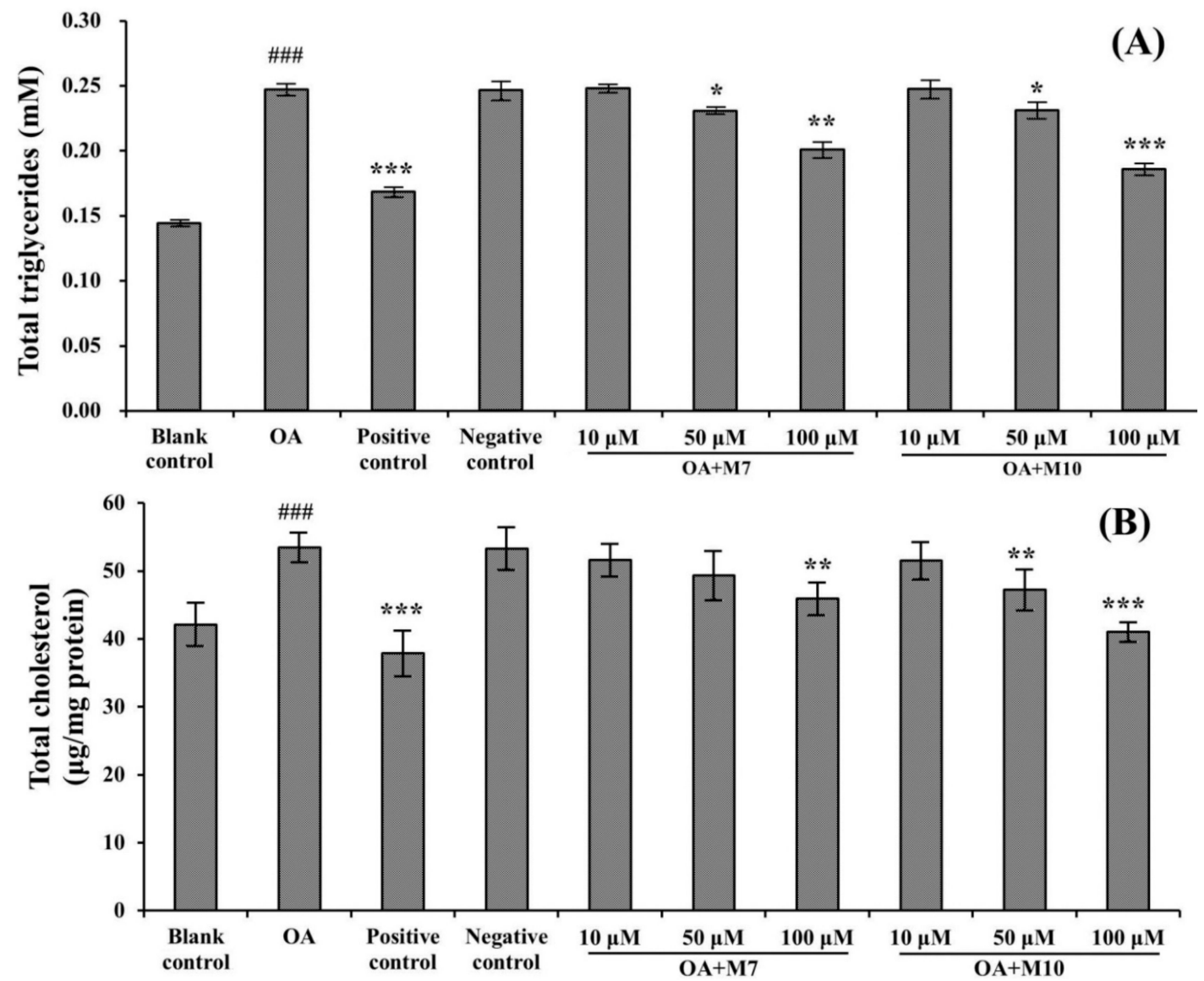

Figure 4. Effects of VIAPW (M7) and IRWWW (M10) from miiuy croaker (M. miiuy) muscle on the intracellular contents of total triglycerides (TG) (A) and cholesterol (TC) (B) in HepG2 cells at the concentrations of 10, 50, and $100 \mu \mathrm{m}$. Simvastatin (SV) and FMPLH (M5) were used as the positive control and negative control, respectively. Means \pm SD $(n=3)$ are used to express the experiment data. \#\#\# $p<0.001$ versus control group; ${ }^{* * *} p<0.001,{ }^{* *} p<0.01,{ }^{*} p<0.05$ versus OA model and negative control groups.

\subsection{Effects of VIAPW (M7) and IRWWW (M10) on the Expression Levels of Genes Involved in Lipid Metabolism}

Lipid metabolism refers to the oxidation and synthesis of fats in cells, which involve the breaking down or storage of fats for energy. The adenosine $5^{\prime}$-monophosphate (AMP)-activated protein kinase (AMPK) pathway plays a major role for regulating the lipid metabolism and is a key sensor of energy homeostasis at the cell level [33]. Previous studies confirmed that AMPK could phosphorylate and inactivate the functions of acetyl-CoA carboxylase (ACC), SREBP, HMGR, and FAS and then control the biosynthesis of TG and sterols [24]. In addition, AMPK activation can increase the expression of proteins associated with FA oxidation, including carnitine palmitoyltransferase I (CPT-1), PPARs, and acyl-CoA oxidase 1 (ACOX-1) [34].

Effects of VIAPW (M7) and IRWWW (M10) on the expression levels of genes drawn into lipid synthesis (SREBP-1c, SREBP-2, FAS, ACC, and HMGR) and oxidation (PPAR $\alpha$, ACOX-1, and CPT-1) in HepG2 cells were investigated, and the data were analyzed using the qRT-PCR method (Figure 5). In comparison with the OA model group, VIAPW (M7) could extremely significantly decrease the expression levels of genes of SREBP-1c $(p<0.001)$, very significantly reduce the expression levels of genes of ACC and FAS $(p<0.01)$, and significantly decrease the expression levels of genes of SREBP-2 and HMGR $(p<0.05)$. In addition, Figure 6 indicated that VIAPW (M7) could significantly increase the expression levels of genes of PPAR $\alpha$, ACOX-1, and CPT- $1(p<0.05)$. 

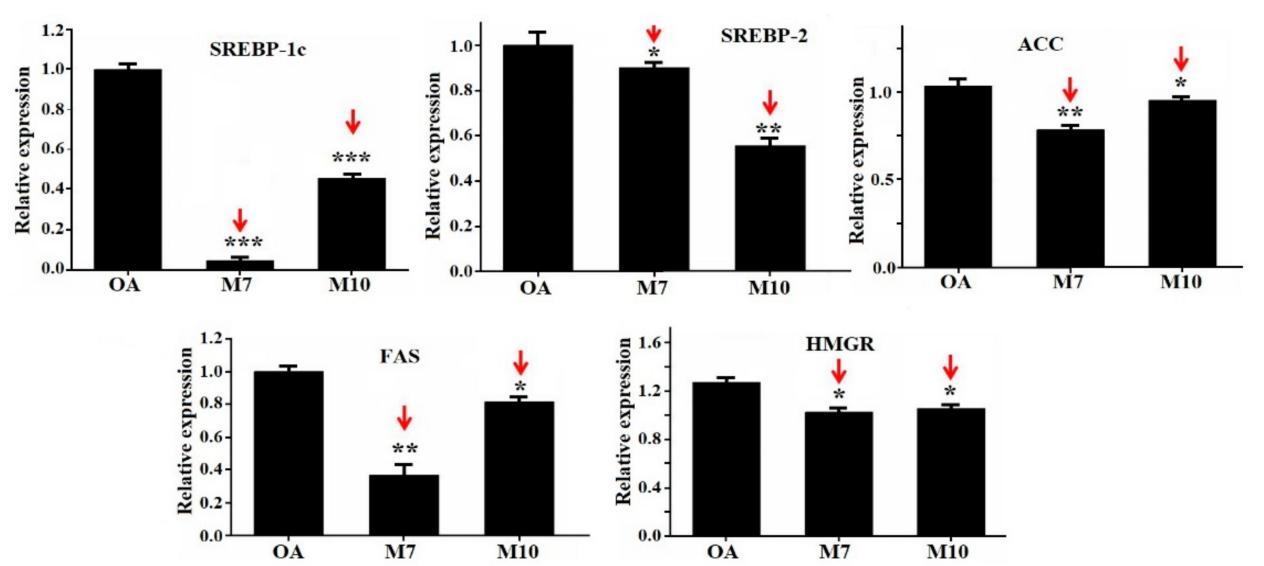

Figure 5. Effects of VIAPW (M7) and IRWWW (M10) from miiuy croaker (M. miiuy) muscle at a concentration of $100 \mu \mathrm{m}$ on the expression levels of genes involved in lipid synthesis (SREBP-1c, SREBP-2, FAS, ACC, and HMGR) in HepG2 cells. Means \pm SD $(n=3)$ are used to express the experiment data. ${ }^{* * *} p<0.001,{ }^{* *} p<0.01,{ }^{*} p<0.05$ versus OA model group.
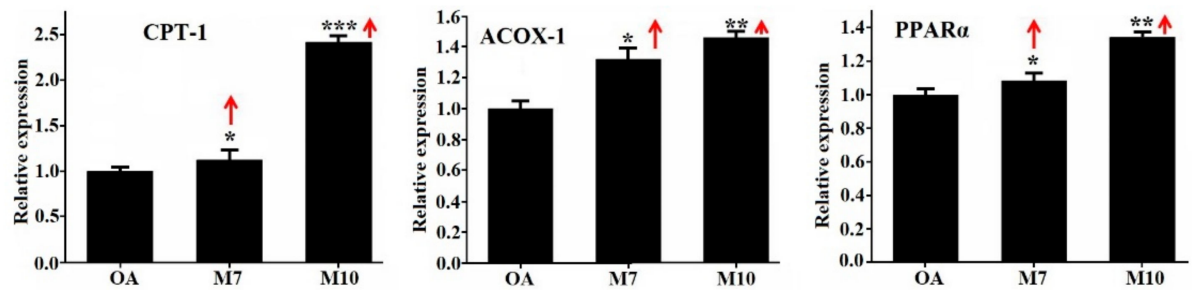

Figure 6. Effects of VIAPW (M7) and IRWWW (M10) from miiuy croaker (M. miiuy) muscle on the expression levels of genes involved in lipid synthesis (SREBP-1c, SREBP-2, FAS, ACC, and HMGR) and oxidation (PPAR $\alpha$, ACOX-1, and CPT- 1$)$ in HepG2 cells. Means \pm SD $(n=3)$ are used to express the experiment data. ${ }^{* *} p<0.001,{ }^{* *} p<0.01,{ }^{*} p<0.05$ versus OA model group.

The effect of IRWWW (M10) on the expression levels of genes related to the lipid metabolism showed a similar tendency with VIAPW (M7), but the expression levels of genes were slightly different from those of VIAPW (M7). Compared with the OA model group, IRWWW (M10) could extremely significantly down-regulate the expression level of SREBP-1c gene $(p<0.001)$, very significantly down-regulate the expression levels of SREBP-2 genes $(p<0.01)$, and significantly down-regulate the expression levels of genes of ACC, FAS, and HMGR $(p<0.05)$. Moreover, IRWWW (M10) extremely significantly up-regulated the expression level of gene of CPT-1 $(p<0.001)$ and very significantly up-regulated the expression levels of genes of PPAR $\alpha$ and ACOX-1 $(p<0.01)$.

Lipid accumulation in hepatic cells could be induced through increasing de novo lipogenesis and lipid intake and reducing lipid $\beta$-oxidation. SREBP-1c and SREBP-2 are the preponderant isoforms and control the levels of ACC and HMGR in hepatic cells, which were the first enzymes recognized as downstream targets for AMPK and key enzymes in FA and TC synthesis [35]. SREBP-1c is a critical transcription factor for increasing the expression levels of lipogenesis genes and plays a significant part in the pathogenesis of hepatic steatosis [36,37]. SREBP-1c can up-regulate the expression level of lipogenic enzymes of ACC, which is responsible for the biosynthesis of malonyl-CoA as the rate-controlling enzyme. Malonyl-CoA is an essential precursor in the synthesis of FA and increases FAS. In addition, malonyl-CoA is a powerful suppressor of mitochondrial lipid oxidation through regulating the rate-limiting enzyme of CPT-1 in FA oxidation, whereas SREBP-2 was generally regarded to be involved in TC synthesis by regulating the gene expression level of HMGR, which limits the biosynthesis of TC and catalyzes the conversion of HMG-CoA into mevalonate. Down-regulating the expression level of the gene of HMGR can lead to the rapidly reducing cholesterol biosynthesis [35]. As a nuclear receptor, PPAR $\alpha$ highly expresses in liver and plays a crucial role in the regulation of lipid metabolism [26]. Activation of PPAR $\alpha$ can increase the expressions levels of genes drawn 
into FA oxidation and transport, such as FA transport protein (FATP) and CPT-1, which lead to an increased oxidation of TG and FA and decreased synthesis of TG and FA [38]. Our results revealed that VIAPW (M7) and IRWWW (M10) could prevent OA-induced hepatic lipid accumulation through down-regulating the expression level of gene of SREBP-1C to inhibit fat synthesis and up-regulating the expression of gene of PPAR $\alpha$ to promote lipid oxidation in HepG2 cells.

\subsection{Effects of VIAPW (M7) and IRWWW (M10) on the Expression Levels of Proteins Related to Lipid Metabolism in HepG2 Cells}

Western blotting is a key experimental technique used in molecular biology and immunogenetics to test the specific proteins in a complicated mixture prepared from cells. For proving the results of qRT-PCR, we applied the western blot assay to detect the expression levels of proteins including SREBP-1c and ACC related to lipid biosynthesis. As shown in Figure 7, VIAPW (M7) and IRWWW (M10) could very significantly $(p<0.01)$ and significantly $(p<0.05)$ down-regulate the expression levels of protein of SREBP-1c, respectively. In addition, VIAPW (M7) and IRWWW (M10) could significantly $(p<0.05)$ and very significantly $(p<0.01)$ down-regulate the expression levels of protein of ACC, respectively.

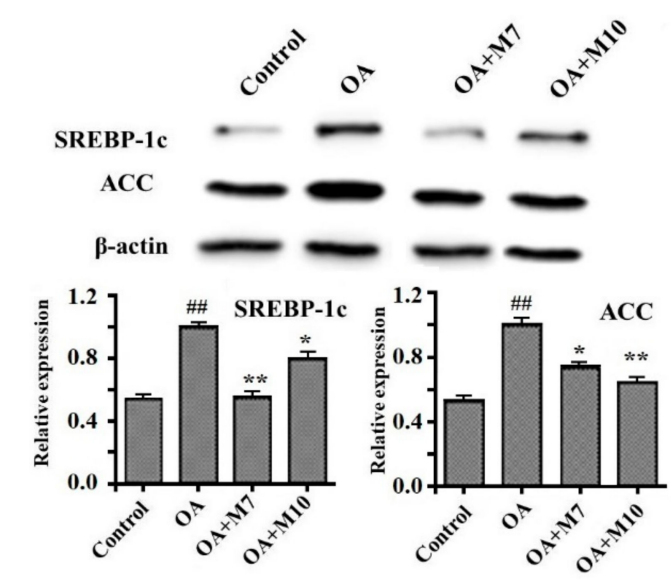

Figure 7. Effects of VIAPW (M7) and IRWWW (M10) from miiuy croaker (M. miiuy) muscle on the expression levels of proteins of SREBP-1c and ACC associated with lipid metabolism in HepG2 cells. Means $\pm \mathrm{SD}(\mathrm{n}=3)$ are used to express the experiment data. ${ }^{\# \#} p<0.01$ versus normal control group; ** $p<0.01$ and * $p<0.05$ versus OA model group.

Hepatic lipid accumulation might be induced by increasing lipid synthesis and/or decreasing lipid catabolism $[39,40]$. As a critical transcription factor, SREBP-1c can stimulate the expression levels of lipogenic enzymes (ACC and FAS) involved in lipid synthesis [36]. ACC is a rate-limiting enzyme for the synthesis of malonyl-CoA, which is a key precursor of FA biosynthesis and a powerful depressor of FA oxidation in mitochondria [35]. Western blotting results proved that VIAPW (M7) and IRWWW (M10) could significantly down-regulate the expression levels of proteins of SREBP-1c and ACC, which further decreased the biosynthesis of FA and increased the oxidation of mitochondrial FA. The results were in agreement with the effects of VIAPW (M7) and IRWWW (M10) on the expression levels of genes drawn into lipid metabolism in HepG2 cells.

\section{Conclusions}

In summary, the present results proved that VIAPW and IRWWW from protein hydrolysate of miiuy croaker (M. miiuy) muscle could play an important role in decreasing OA-induced intracellular lipid accumulation in HepG2 cells. Their hypolipidemic mechanism was to regulate the AMPK signaling pathway, which further down-regulated the expression levels of lipogenesis genes and up-regulated the expression levels of lipolysis genes. These results provide an important basis for 
developing VIAPW and IRWWW as natural additive in functional products for prophylaxis and adjuvant cure of liver-related hyperlipidemia diseases.

Author Contributions: B.W. and C.-F.C. conceived and designed the experiments. Y.-M.W., X.P. and Y.H. performed the experiments and analyzed the data. C.-F.C. and B.W. contributed the reagents, materials, and analytical tools and wrote the paper. All authors have read and agreed to the published version of the manuscript.

Funding: This work was funded by the National Natural Science Foundation of China (No. 31872547, 81673349) and Zhejiang Province Public Technology Research Project (No. LGN18D060002).

Conflicts of Interest: The authors declare no conflict of interest.

\section{References}

1. Lee, H.; Choi, J.M.; Cho, J.Y.; Kim, T.E.; Lee, H.J.; Jung, B.H. Regulation of endogenic metabolites by rosuvastatin in hyperlipidemia patients: An integration of metabolomics and lipidomics. Chem. Phys. Lipids 2018, 214, 69-83. [CrossRef]

2. Affane, F.; Louala, S.; El Imane Harrat, N.; Bensalah, F.; Chekkal, H.; Allaoui, A.; Lamri-Senhadji, M. Hypolipidemic, antioxidant and antiatherogenic property of sardine by-Products proteins in high-Fat diet induced obese rats. Life Sci. 2018, 199, 16-22. [CrossRef]

3. Kirakosyan, A.; Gutierrez, E.; Ramos Solano, B.; Seymour, E.M.; Bolling, S.F. The inhibitory potential of Montmorency tart cherry on key enzymes relevant to type 2 diabetes and cardiovascular disease. Food Chem. 2018, 252, 142-146. [CrossRef] [PubMed]

4. Mudgil, P.; Kamal, H.; Yuen, G.C.; Maqsood, S. Characterization and identification of novel antidiabetic and anti-Obesity peptides from camel milk protein hydrolysates. Food Chem. 2018, 259, 46-54. [CrossRef] [PubMed]

5. Tacherfiout, M.; Petrov, P.D.; Mattonai, M.; Ribechini, E.; Ribot, J.; Bonet, M.L.; Khettala, B. Antihyperlipidemic effect of a Rhamnus alaternus leaf extract in Triton-Induced hyperlipidemic rats and human HepG2 cells. Biomed. Pharmacother. 2018, 101, 501-509. [CrossRef] [PubMed]

6. Prados, I.M.; Marina, M.L.; García, M.C. Isolation and identification by high resolution liquid chromatography tandem mass spectrometry of novel peptides with multifunctional lipid lowering capacity. Food Res. Int. 2018, 111, 77-86. [CrossRef] [PubMed]

7. Aiello, G.; Ferruzza, S.; Ranaldi, G.; Sambuy, Y.; Arnoldi, A.; Vistoli, G.; Lammi, C. Behavior of three hypocholesterolemic peptides from soy protein in an intestinal model based on differentiated Caco-2 cell. J. Funct. Foods 2018, 45, 363-370. [CrossRef]

8. Song, D.; Jiang, J. Hypolipidemic Components from Medicine Food Homology Species Used in China: Pharmacological and Health Effects. Arch. Med. Res. 2017, 48, 569-581. [CrossRef]

9. Jemil, I.; Abdelhedi, O.; Nasri, R.; Mora, L.; Marrekchi, R.; Jamoussi, K.; ElFeki, A.; Hajji, M.; Toldrá, F.; Nasri, M. Hypolipidemic, antiobesity and cardioprotective effects of sardinelle meat flour and its hydrolysates in high-Fat and fructose diet fed Wistar rats. Life Sci. 2017, 176, 54-66. [CrossRef]

10. Zhao, Y.Q.; Zhang, L.; Tao, J.; Chi, C.F.; Wang, B. Eight antihypertensive peptides from the protein hydrolysate of Antarctic krill (Euphausia superba): Isolation, identification, and activity evaluation on human umbilical vein endothelial cells (HUVECs). Food Res. Int. 2019, 121, 197-204. [CrossRef]

11. Sila, A.; Bougatef, A. Antioxidant peptides from marine by-products: Isolation, identification and application in food systems. A review. J. Funct. Foods 2016, 21, 10-26. [CrossRef]

12. Chi, C.F.; Hu, F.Y.; Wang, B.; Li, T.; Ding, G.F. Antioxidant and anticancer peptides from protein hydrolysate of blood clam (Tegillarca granosa) muscle. J. Funct. Foods 2015, 15, 301-313. [CrossRef]

13. Qiu, Y.T.; Wang, Y.M.; Yang, X.R.; Zhao, Y.Q.; Chi, C.F.; Wang, B. Gelatin and antioxidant peptides from gelatin hydrolysate of skipjack tuna (Katsuwonus pelamis) scales: Preparation, identification and activity evaluation. Mar. Drugs 2019, 17, 565. [CrossRef] [PubMed]

14. Zhang, L.; Zhao, G.X.; Zhao, Y.Q.; Qiu, Y.T.; Chi, C.F.; Wang, B. Identification and active evaluation of antioxidant peptides from protein hydrolysates of skipjack tuna (Katsuwonus pelamis) head. Antioxidants 2019, 8, 318. [CrossRef]

15. Fan, X.; Cui, Y.; Zhang, R.; Zhang, X. Purification and identification of anti-Obesity peptides derived from Spirulina platensis. J. Funct. Foods 2018, 47, 350-360. [CrossRef] 
16. Pak, V.V.; Koo, M.; Kwon, D.Y.; Yun, L. Design of a highly potent inhibitory peptide acting as a competitive inhibitor of HMG-CoA reductase. Amino Acids 2012, 43, 2015-2025. [CrossRef]

17. Nagaoka, S.; Futamura, Y.; Miwa, K.; Awano, T.; Yamauchi, K.; Kanamaru, Y.; Tadashi, K.; Kuwata, T. Identification of novel hypocholesterolemic peptides derived from bovine milk $\beta$-Lactoglobulin. Biochem. Biophys. Res. Commun. 2001, 281, 11-17. [CrossRef]

18. Lee, Y.G.; Cho, J.Y.; Hwang, E.J.; Jeon, T.I.; Moon, J.H. Glu-Phe from onion (Allium Cepa L.) attenuates lipogenesis in hepatocytes. Biosci. Biotechnol. Biochem. 2017, 81, 1409-1416. [CrossRef]

19. Shi, W.; Hou, T.; Liu, W.; Guo, D.; He, H. The hypolipidemic effects of peptides prepared from Cicer arietinum in ovariectomized rats and HepG2 cells. J. Sci. Food Agric. 2019, 99, 576-586. [CrossRef]

20. Wang, B.; Li, L.; Chi, C.F.; Ma, J.H.; Luo, H.Y.; Xu, Y.F. Purification and characterisation of a novel antioxidant peptide derived from blue mussel (Mytilus edulis) protein hydrolysate. Food Chem. 2013, 138, 1713-1719. [CrossRef]

21. Zhao, W.H.; Luo, Q.B.; Pan, X.; Chi, C.F.; Sun, K.L.; Wang, B. Preparation, identification, and activity evaluation of ten antioxidant peptides from protein hydrolysate of swim bladders of miiuy croaker (Miichthys miiuy). J. Funct. Foods 2018, 47, 503-511. [CrossRef]

22. Yang, X.R.; Zhang, L.; Zhao, Y.Q.; Chi, C.F.; Wang, B. Purification and characterization of antioxidant peptides derived from protein hydrolysate of the marine bivalve mollusk Tergillarca granosa. Mar. Drugs 2019, 17, 251. [CrossRef] [PubMed]

23. He, Y.; Pan, X.; Chi, C.F.; Sun, K.L.; Wang, B. Ten new pentapeptides from protein hydrolysate of miiuy croaker (Miichthys miiuy) muscle: Preparation, identification, and antioxidant activity evaluation. LWT-Food Sci. Technol. 2019, 105, 1-8. [CrossRef]

24. Zhang, Y.; Chen, S.; Wei, C.; Chen, J.; Ye, X. Proanthocyanidins from Chinese bayberry (Myrica rubra Sieb. et Zucc.) leaves regulate lipid metabolism and glucose consumption by activating AMPK pathway in HepG2 cells. J. Funct. Foods 2017, 29, 217-225. [CrossRef]

25. Pan, X.; Zhao, Y.Q.; Hu, F.Y.; Chi, C.F.; Wang, B. Anticancer activity of a hexapeptide from skate (Raja porosa) cartilage protein hydrolysate in HeLa cells. Mar. Drugs 2016, 14, 153. [CrossRef]

26. Madushani Herath, K.H.I.N.; Cho, J.; Kim, A.; Eom, T.K.; Kim, J.S.; Kim, J.B.; Doh, Y.H.; Jee, Y. Phenolic acid and flavonoid-Rich fraction of Sasa quelpaertensis Nakai leaves prevent alcohol induced fatty liver through AMPK activation. J. Ethnopharmacol. 2018, 224, 335-348. [CrossRef]

27. Gerets,H.H.; Tilmant, K.; Gerin, B.; Chanteux,H.; Depelchin, B.O.; Dhalluin, S.; Atienzar, F.A. Characterization of primary human hepatocytes, HepG2 cells, and HepaRG cells at the mRNA level and CYP activity in response to inducers and their predictivity for the detection of human hepatotoxins. Cell Biol. Toxicol. 2012, 28, 69-87. [CrossRef]

28. Pardo Andreu, G.L.; Reis, F.H.; Dalalio, F.M.; Nuñez Figueredo, Y.; Cuesta Rubio, O.; Uyemura, S.A.; Curti, C.; Alberici, L.C. The cytotoxic effects of brown Cuban propolis depend on the nemorosone content and may be mediated by mitochondrial uncoupling. Chem.-Biol. Interac. 2015, 228, 28-34. [CrossRef]

29. Liu, Q.; Bengmark, S.; Qu, S. The role of hepatic fat accumulation in pathogenesis of non-Alcoholic fatty liver disease (NAFLD). Lipids Health Dis. 2010, 9, 42. [CrossRef]

30. Yu, Q.; Liu, Y.; Wu, Y.; Chen, Y. Dihydrocurcumin ameliorates the lipid accumulation, oxidative stress and insulin resistance in oleic acid-induced L02 and HepG2 cells. Biomed. Pharmacother. 2018, 103, 1327-1336. [CrossRef]

31. Ren, R.; Gong, J.; Zhao, Y.; Zhuang, X.; Ye, Y.; Huang, F.; Lin, W. Sulfated polysaccharide from Enteromorpha prolifera suppresses SREBP-1c and ACC expression to lower serum triglycerides in high-Fat diet-Induced hyperlipidaemic rats. J. Funct. Foods 2018, 40, 722-728. [CrossRef]

32. Sozen, E.; Ozer, N.K. Impact of high cholesterol and endoplasmic reticulum stress on metabolic diseases: An updated mini-Review. Redox Bio. 2017, 12, 456-461. [CrossRef] [PubMed]

33. Day, E.A.; Ford, R.J.; Steinberg, G.R. AMPK as a therapeutic target for treating metabolic diseases. Trends Endocrin. Met. 2017, 28, 545-560. [CrossRef] [PubMed]

34. Randy, A.; Kim, M.; Nho, C.W. Ligularia fischeri and its constituent 3,4-Dicaffeoylquinic acid improve obesity-Induced nonalcoholic fatty liver disease by regulating lipid metabolism and activating AMPK. J. Funct. Foods 2016, 27, 1-16. [CrossRef] 
35. Liu, X.; Hao, J.J.; Zhang, L.J.; Zhao, X.; He, X.X.; Li, M.M.; Zhao, X.L.; Wu, J.D.; Qiu, P.J.; Yu, G.L. Activated AMPK explains hypolipidemic effects of sulfated low molecular weight guluronate on HepG2 cells. Eur. J. Med. Chem. 2014, 85, 304-310. [CrossRef] [PubMed]

36. Ao, N.; Yang, J.; Wang, X.; Du, J. Glucagon-Like peptide-1 preserves non-Alcoholic fatty liver disease through inhibition of the endoplasmic reticulum stress associated pathway. Hepatol. Res. 2016, 46, 343-353. [CrossRef] [PubMed]

37. Yun, Y.R.; Kim, J.H.; Kim, J.H.; Jung, M.H. Protective effects of gomisin N against hepatic steatosis through AMPK activation. Biochem. Biophys. Res. Commun. 2017, 482, 1095-1101. [CrossRef]

38. Liu, B.; Yang, T.; Luo, Y.; Zeng, L.; Shi, L.; Wei, C.; Nie, Y.; Cheng, Y.; Lin, Q.; Luo, F. Oat $\beta$-Glucan inhibits adipogenesis and hepatic steatosis in high fat diet induced hyperlipidemic mice via AMPK signalling. J. Funct. Foods 2018, 41, 72-82. [CrossRef]

39. Kabirifar, R.; Ghoreshi, Z.; Rezaifar, A.; Binesh, F.; Bamdad, K.; Moradi, A. Curcumin, quercetin and atorvastatin protected against the hepatic fibrosis by activating AMP-Activated protein kinase. J. Funct. Foods 2018, 40, 341-348. [CrossRef]

40. Li, T.; Wen, L.; Cheng, B. Cordycepin alleviates hepatic lipid accumulation by inducing protective autophagy via PKA/mTOR pathway. Biochem. Biophys. Res. Commun. 2019, 516, 632-638. [CrossRef]

(C) 2020 by the authors. Licensee MDPI, Basel, Switzerland. This article is an open access article distributed under the terms and conditions of the Creative Commons Attribution (CC BY) license (http://creativecommons.org/licenses/by/4.0/). 